\title{
ORQUÍDEAS SILVESTRES COMERCIALIZADAS EN UN MERCADO TRADICIONAL DE CHIAPAS, MÉXICO
}

\author{
WILD ORCHIDS TRADED IN A TRADITIONAL MARKET IN CHIAPAS, MEXICO \\ Derio antonio Jiménez-López ${ }^{1 *}$, Eduardo Alberto Pérez-García², Nayely Martínez-Meléndez ${ }^{3}$ y Rodolfo \\ SOLANO ${ }^{4}$
}

\begin{abstract}
${ }^{1}$ Centro del Cambio Global y la Sustentabilidad A.C. (CCGS), Villahermosa, Tabasco, México.
${ }^{2}$ Departamento de Ecología y Recursos Naturales, Facultad de Ciencias, Universidad Nacional Autónoma de México, Ciudad de México, México.

${ }^{3}$ Departamento de Conservación de la Biodiversidad, El Colegio de la Frontera Sur, San Cristóbal de las Casas, Chiapas, México. ${ }^{4}$ Instituto Politécnico Nacional, Centro Interdisciplinario de Investigación para el Desarrollo Integral Regional Unidad Oaxaca, Oaxaca, México.
\end{abstract}

*Autor de correspondencia: derio.jimenezlopez@gmail.com

\begin{abstract}
Resumen
Antecedentes: La comercialización de orquídeas es una práctica común en los mercados mexicanos, especialmente en regiones de alta biodiversidad.

Preguntas y/o hipótesis: ¿Cuántas especies de orquídeas se vendieron en el mercado de Las Margaritas? ¿De dónde se extrajeron? ¿Su disponibilidad (riqueza) mostró una variación temporal a lo largo de un año? ¿Cuál fue la relación entre la vistosidad de las flores (tamaño) y su precio de venta?

Especies en estudio: Familia Orchidaceae.

Sitio de estudio y fechas: Mercado de Las Margaritas, Chiapas, 2014.

Métodos: Entrevistamos a 15 vendedores del mercado (12 mujeres y tres hombres). Determinamos todas las especies comercializadas, su lugar de extracción, y analizamos la relación entre la vistosidad de cada flor y su precio de venta, mediante un análisis de correlación.

Resultados: Se registraron 60 orquídeas en venta. Cuatro de éstas, estaban en alguna categoría de riesgo (NOM-059- SEMARNAT-2010): Lycaste skinneri, Laelia superbiens, Cuitlauzina pulchella y Oncidium leucochilum. El período de mayor venta fue de diciembre a enero, mientras que el más bajo fue de septiembre a octubre. La mayoría de las orquídeas fueron extraídas de la Región Terrestre Prioritaria El Momón-Montebello. La vistosidad se relacionó positivamente con precios de venta más altos $(r=0,67, p<0,01)$.

Conclusiones: Se observó una relación significativa entre la vistosidad de la flor y el precio de venta. La comercialización de orquídeas se realizó durante todo el año; sin embargo, mostró dos picos altos (entre diciembre - enero y mayo - junio). Por lo tanto, es necesario organizar talleres con los vendedores para discutir la posibilidad de continuar esta actividad bajo un marco legal regulado.
\end{abstract}

Palabras clave: Comercio ilegal, mercados tradicionales, Orchidaceae, Región Terrestre Prioritaria El Momón-Montebello.

\begin{abstract}
Background: Orchid commercialization is a common practice in Mexican markets, especially in high biodiversity regions.

Question / Hypothesis: How many orchid species were sold in the Las Margaritas Market? From where were they extracted? Did their availability (richness) show a temporal variation along of one year? Which was the relation between the showiness of the flowers (size) and their selling price?

Studied species: Orchidaceae family.

Study site and years of study: Las Margaritas Market, Chiapas, 2014.

Methods: We interviewed 15 market-sellers (12 women and three men). We determined all the commercialized species, their place of extraction and the relationship between the showiness of each flower (flower size) and its selling price, through a correlation analysis.

Results: Sixty orchid species were registered on sale. Four of these species were under an at-risk category (NOM-059- SEMARNAT-2010): Lycaste skinneri, Laelia superbiens, Cuitlauzina pulchella and Oncidium leucochilum. The highest selling period was December-January, while the lowest was September-October. Most of the orchids were extracted from the Priority Terrestrial Region El Momón-Montebello. The showiness of each individual was positively with higher selling prices $(r=0.67, p<0.01)$.

Conclusions: A significant relation was observed between the flower showiness and selling price. Orchid commercialization was performed during all the year; however, it showed two high peaks (December-January and May-June). Thus, it is necessary to organize workshops with the sellers to discuss the possibility of continuing this activity under a regulated legal framework.

Keywords: Illegal trade, Orchidaceae, Priority Terrestrial Region El Momón-Montebello, Traditional markets.
\end{abstract}

This is an open access article distributed under the terms of the Creative Commons Attribution License CCBY-NC (4.0) international. https://creativecommons.org/licences/by-nc/4.0/ 
Los mercados tradicionales son centros donde se comercializan productos de valor agronómico y forestal nativos de las regiones donde se establecen, constituyendo una fuente importante para el conocimiento de la biodiversidad local y de la cultura de diversos grupos rurales e indígenas (Bye \& Linares 1983). En Mesoamérica, estos mercados tienen origen precolombino y se caracterizan por la interacción de diferentes grupos étnicos y por ser intermitentes, comúnmente existe un día de "tianguis", palabra que deriva del náhuatl y se refiere a un mercado tradicional que se instala en un lugar público un día a la semana (Arellanes et al. 2013). Actualmente interactúan con los mercados modernos (Shackleton 2001, Arellanes et al. 2013, Farfán-Heredia et al. 2018). Los mercados tradicionales generan ingresos substanciales a la economía familiar de los vendedores (Bye \& Linares 1983, Reuter 2009, Vodouhê et al. 2009) y son lugares importantes para la socialización (Linares \& Bye 2016). Dentro de los productos regionales que más se venden o intercambian en estos mercados están los alimentos (animales domésticos, productos de la milpa, frutas de temporada, café, etc.), artesanías, utensilios de cocina, aditamentos para el trabajo rural, plantas medicinales y diversos productos forestales no maderables (PFNM). Muchos de estos productos incluyen el comercio de especies silvestres (Linares \& Bye 2016, Villamar 2016.) que, en la mayoría de los casos, son comercializados a partir de ejemplares extraídos de su hábitat, sin un plan de manejo o el empleo de técnicas para un aprovechamiento racional. A pesar de la importancia de estos mercados para la subsistencia de las comunidades rurales y la alta frecuencia del comercio de vida silvestre en ellos, se conocen muy pocos estudios sobre la dependencia de los vendedores por una actividad informal que se realiza fuera de un marco legal. El entendimiento de la problemática asociada a la condición social de los vendedores, su dependencia económica por el comercio de vida silvestre, así como el origen, disponibilidad y riqueza de los recursos biológicos comercializados, puede contribuir con información importante para el diseño de planes de aprovechamiento para las especies afectadas por esta práctica (Mondragón 2009, Rojas-Méndez et al. 2017).

En México crecen un poco más de 1,300 orquídeas silvestres, pertenecientes a 168 géneros (Hágsater et al. 2005), por lo que es la tercera familia de mayor riqueza en el país, superadas únicamente por Asteraceae y Fabaceae (Villaseñor 2016). Lamentablemente, la orquideoflora mexicana enfrenta problemas de conservación, debido a que la distribución de su mayor riqueza coincide con las regiones donde se presentan mayores tasas de deforestación y cambio de uso de suelo, así como comercio ilícito de vida silvestre (Soto-Arenas et al. 2007, Solano-Gómez et al. 2012). En México, el comercio ilícito de orquídeas silvestres existe, porque hay un efecto de oferta-demanda, el cual se incrementa cuando el periodo de floración de las especies de valor ornamental comercializadas coincide con algunas festividades religiosas, como Semana Santa, Día de Muertos y Navidad (Halbinger \& Soto 1997, Flores-Palacios \& Valencia-Díaz 2007, Solano-Gómez et al. 2010, Cruz-García et al. 2015, Emeterio-Lara et al. 2016, Jiménez-López et al. 2019).

Sobre lo que se ha podido estudiar en torno a la problemá- tica del comercio ilícito de orquídeas y otras epífitas vasculares en México, se reporta que esta actividad, básicamente, es realizada por mujeres (Flores-Palacios \& Valencia-Díaz 2007, Arellanes et al. 2013, Cruz-García et al. 2015), y que los vendedores de mayor edad, hablantes de una lengua indígena y con una escolaridad nula o escasa (indicativos de su pobreza) obtienen menos ingresos en comparación con los vendedores más jóvenes y con mayor escolaridad (CruzGarcía et al. 2015). Por otro lado, aun cuando el mercado incluya disponibilidad de orquídeas con relación a la oferta y demanda, las ganancias se concentran en unas cuantas especies de valor ornamental o ceremonial (Cruz-García et al. 2015, Emeterio-Lara et al. 2016, Rojas-Méndez et al. 2017, Jiménez-López et al. 2019).

La legislación ambiental de México contempla la protección de las orquídeas nativas y considera que las poblaciones campesinas e indígenas, poseedoras de los bosques donde éstas crecen, pueden ser beneficiarios de un aprovechamiento basado en estas plantas, siempre que cuenten con el permiso de la autoridad ambiental, la SEMARNAT (SEMARNAT 2014). La regulación para el aprovechamiento de orquídeas está contemplada en la Ley General de Vida Silvestre (LGVS; SEMARNAT 2018a) y la Ley General de Desarro1lo Forestal Sustentable (LGDFS; SEMARNAT 2018b). De acuerdo con la LGVS, para autorizar el aprovechamiento de una o varias de las 181 orquídeas consideradas en riesgo e incluidas en la NOM-059-SEMARNAT-2010 (SEMARNAT 2010), se requiere la elaboración de un plan de manejo y el registro de una Unidad de Manejo Ambiental (UMA), aprobados por la SEMARNAT. El aprovechamiento para orquídeas no consideradas en riesgo, está regulado por la LGDFS y requiere de un aviso por escrito y un programa de manejo simplificado autorizados por la SEMARNAT. Por otro lado, la NOM-005-SEMARNAT-1997 (SEMARNAT 2003) establece las procedimientos, criterios y especificaciones para un aprovechamiento de orquídeas, siempre que no se incluyan especies en riesgo. En todos los casos, la autoridad ambiental debe realizar acciones de vigilancia para verificar el correcto cumplimiento del aprovechamiento y avalar su continuidad. Sin embargo, en el país no se conocen experiencias donde, para el aprovechamiento de orquídeas, a través de su comercio en mercados tradicionales, se cumpla con la regulación arriba mencionada, pues se realiza a partir de la extracción de ejemplares silvestres y sin permisos de la autoridad ambiental, por lo que resulta ser una actividad ilegal.

En el presente trabajo se analiza la relación entre la vistosidad de las flores con el precio de venta y se describe la variación temporal de la riqueza a lo largo de un año y las zonas de extracción del comercio de orquídeas en el mercado municipal Juan Sabines Guerrero de Las Margaritas. La mayor proporción del territorio de este municipio se encuentra dentro de la Región Terrestre Prioritaria (RTP) conocida como El Momón-Montebello (Arriaga-Cabrera et al. 2000), una región importante debido a que ahí se encuentran cinco tipos de vegetación: bosque tropical perennifolio, bosque mesófilo de montaña, bosque de pino, bosque de pino-encino y bosque de pino-encino-liquidámbar (Rzedowski 1978). Alberga la mayor riqueza de orquídeas en México (Soto-Arenas 2001) 
y una gran cantidad de epífitas vasculares en bosque mesófilo de montaña dominado por Podocarpus, por todo esto es considerada prioritaria para la conservación biológica en el país (Arriaga-Cabrera et al. 2000). En un estudio preliminar (Jiménez-López et al. 2015), se presentó la lista de orquídeas comercializadas en este mercado, pero ahora se aporta mayor información, con la finalidad de: (1) determinar la variación temporal de la riqueza de las orquídeas comercializadas en el mercado a lo largo de un año, (2) identificar las zonas de extracción de orquídeas para su venta en el mercado y (3) analizar la relación entre la vistosidad de las flores (tamaño) con el precio de venta de las orquídeas comercializadas, considerando la hipótesis de que cuanto más grande es la flor de una especie, mayor es su precio de venta.

\section{Materiales y métodos}

Área de estudio. El trabajo se realizó en el municipio de Las Margaritas, Chiapas, el cual forma parte de las regiones fisiográficas montañas de oriente y altos de Chiapas (Müllerried 1957). Limita al norte con los municipios de Altamirano y Ocosingo, al este con Maravilla Tenejapa, al sur con la República de Guatemala, la Trinitaria y la Independencia y al oeste con Comitán de Domínguez y Chanal, con una altitud de 1,520 m snm. (SEGOB 2010). Esta investigación se centró en el mercado municipal Juan Sabines Guerrero, que se encuentra ubicado en la zona centro de la cabecera municipal de Las Margaritas, y cuyas coordenadas son: $16^{\circ} 18^{\prime} 55^{\prime \prime} \mathrm{N}$ y $91^{\circ} 58^{\prime} 54^{\prime \prime} \mathrm{O}$ (Figura 1).

Recolección de datos. El muestreo se realizó de enero a diciembre de 2014, se realizaron visitas al mercado y sus alrededores, cada dos semanas, el día domingo, en que se instala el tianguis. Se realizaron observaciones directas en los puntos de venta de orquídeas; ahí mismo se hizo la determinación taxonómica de los ejemplares; se fotografiaron aquellos especímenes cuya determinación in situ no fue posible y se consultó a especialistas para su posterior determinación. En el mercado se ubicaron a las personas que venden orquídeas, se les solicitó permiso para preguntarles sobre su actividad, mediante una entrevista semiestructurada (Mondragón 2015). De aproximadamente 29 vendedores, 15 accedieron a responder la entrevista (12 mujeres y 3 hombres), de éstos se obtuvo información sobre su edad, género, lugar donde extraen orquídeas y si realizan otra actividad dentro del mercado para generar ingresos. En algunos casos se recurrió a la técnica de entrevista-compra para obtener el precio de venta de alguna especie (Bye \& Linares 1983). Además, se registró la forma en que cada vendedor lleva sus orquídeas al mercado (planta completa o pseudobulbos con inflorescencias), y el mes de venta. Se consultó la Norma Oficial Mexicana NOM-059-SEMARNAT-2010 (SEMARNAT 2010), para conocer en qué categoría de riesgo se encontraban las especies registradas. No se consultó la lista roja de la IUCN

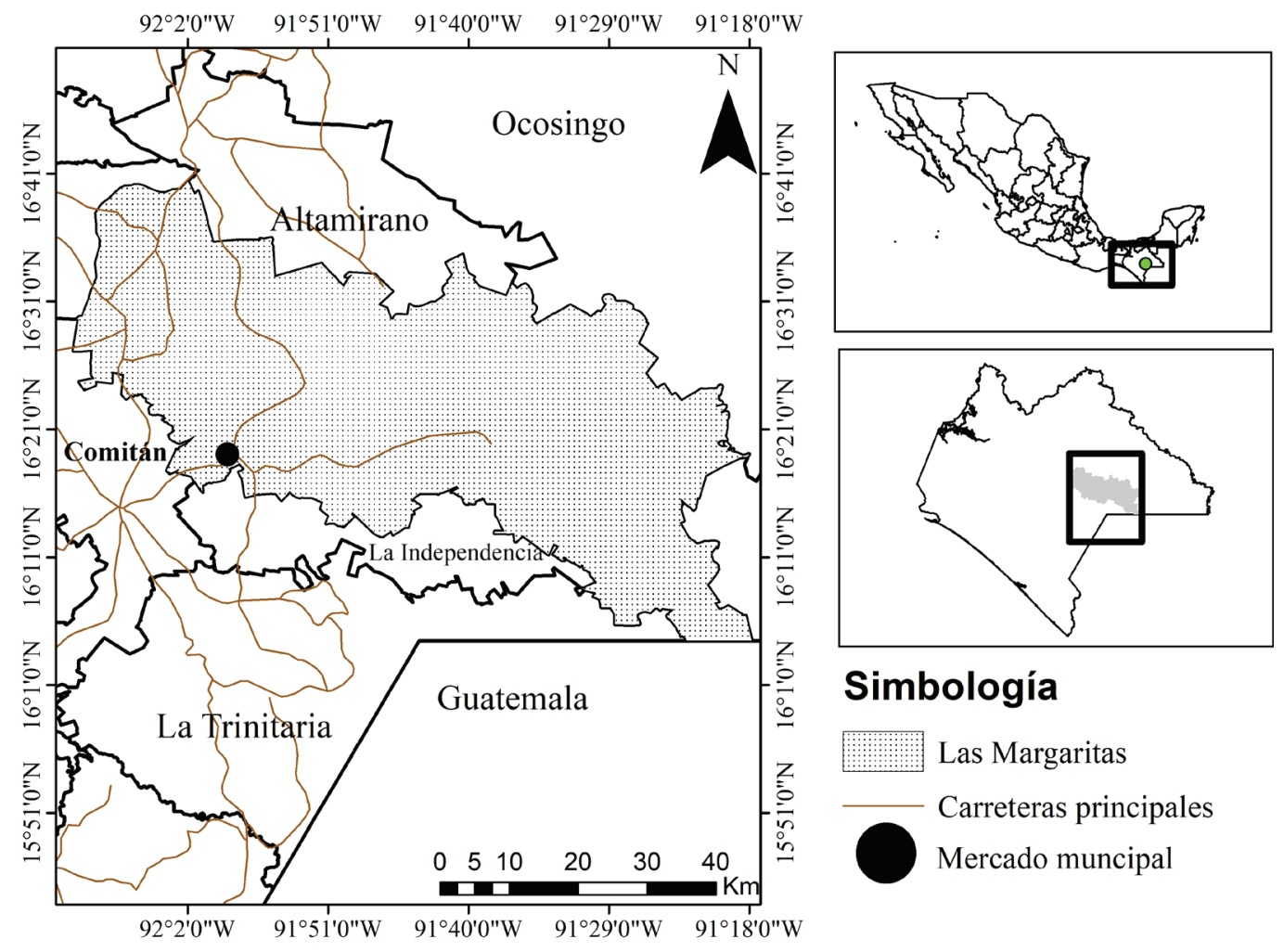

Figura 1. Localización del área de estudio, municipio de Las Margaritas, Chiapas, México. 
debido a que la gran mayoría de especies de orquídeas reportadas para México, no han sido evaluadas y para las que en ella aparecen, no hay garantía de que su evaluación fuera realizada a partir de poblaciones mexicanas.

Análisis de datos. Para reconocer si hay una relación entre la vistosidad de la planta y el precio de venta, se tomó la variable: diámetro de las flores, como la que indica vistosidad. Se realizó un análisis de correlación (prueba de Spearman) para evaluar la colinealidad entre las variables: diámetro de 58 especies que se vendieron vs. precio de venta. Para esta correlación se obtuvo el precio promedio de cada especie en venta, además, para este análisis se omitieron dos especies (Epidendrum sp. y Pleurothallis aff. correllii Luer) debido a que fueron especímenes acompañantes de otras plantas en venta y nunca se vendieron por separado. El análisis fue realizado con la plataforma estadística R (R Core Team 2016).

\section{Resultados}

El comercio de orquídeas en Las Margaritas es claramente local. Los vendedores en su mayoría son agricultores indígenas que viven en los alrededores de la cabecera municipal, donde llevan sus plantas a comercializar. Las orquídeas se colectan en los bosques de sus comunidades, y son compradas por personas de la región, además, este comercio es dominado por mujeres.

A lo largo del año se hicieron 24 visitas al mercado y se registró la venta de un total de 60 especies de orquídeas, de las cuales 52 fueron epífitas y ocho terrestres (Tabla 1); de éstas, solo el $5 \%$ se ofrecían como inflorescencias (Aulosepalum hemichrea (Lindl.) Garay, Bletia purpurea (Lam.) DC y Domingoa purpurea (Lindl.) Van den Berg \& Soto Arenas), $15 \%$ como inflorescencias y plantas completas, y $80 \%$ únicamente como plantas completas. Encyclia Hook. y Epidendrum L. fueron los géneros más comercializados con cinco especies cada uno, seguidos de Oncidium Sw. con cuatro y Maxillaria Ruiz \& Pav., Lycaste Lindl., Pleurothallis R. Br. y Stanhopea Frost ex Hook., con tres cada uno. De estos géneros, solo una especie de Lycaste (L. skinneri Lindl. fue comercializada a mayor precio (\$100 MXN), seguida de Laelia (Laelia superbiens, \$45 MXN) y Mormodes (Mormodes lineata Bateman ex Lindl.; \$45 MXN; Tabla 1).

Del total de especies de orquídeas comercializadas en Las Margaritas, cuatro se encontraron en alguna categoría de riesgo de acuerdo con la legislación mexicana (SEMARNAT 2010): Lycaste skinneri en peligro de extinción, Laelia superbiens, Oncidium endocharis Rchb.f. y Oncidium leucochilum Bateman., como amenazadas (Tabla 1). Las especies con mayor frecuencia de venta durante el año fueron: Laelia anceps Lindl., L. superbiens, Cuitlauzina pulchella (Bateman ex Lindl.) Dressler \& N.H. Williams, Encyclia incumbens (Lindl.) Mabb y Oncidium leucochilum.

Laelia anceps se utiliza para adornar los altares en el Día de Muertos, Cuitlauzina pulchella (Bateman ex Lindl.) Dressler \& N.H. Williams, Epidendrum radicans Pav. ex Lindl, Laelia superbiens y Oncidium leucochilum se utilizan en la época de navidad y Guarianthe aurantiaca (Bateman) Dressler \& W.E. Higgins en la celebración de la Candelaria.

Los meses que registraron mayor número de especies en venta, fueron diciembre y enero con 31 especies (51.66 $\%$ ), seguidos de mayo y junio con 25 especies (41.66\%), en contraparte, septiembre y octubre fueron los meses de menor venta, con seis especies (10\%; Figura 2) y algunas especies fueron registradas en venta en más de un mes en el mercado visitado.

La mayoría de las orquídeas que se comercializan en el mercado provinieron de la RTP El Momón-Montebello (Figura 3); aunque la mayoría de las plantas comercializadas (76 \%) se extrajeron de dos ejidos: Francisco I. Madero (43\%) y Morelia (33.3\%), que se encuentran fuera, pero muy cerca de esta región y son los ejidos más cercanos al mercado mu-

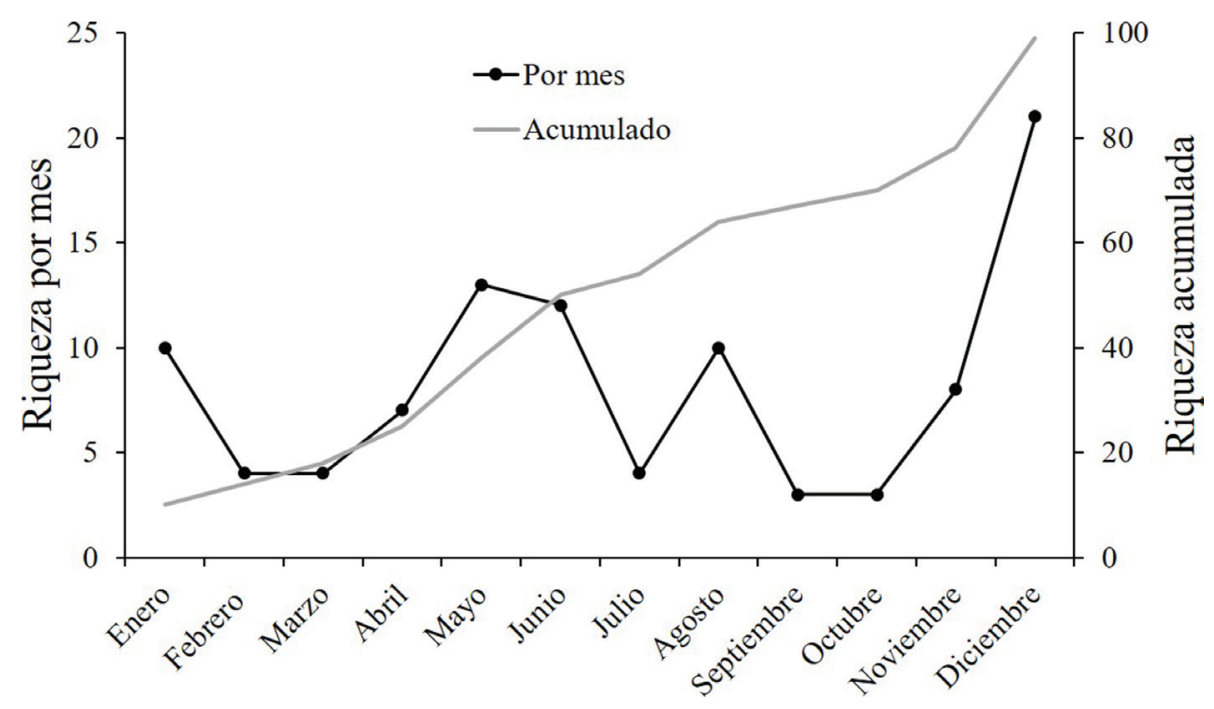

Figura 2. Variación del número de especies en venta en el mercado de Las Margaritas, Chiapas a lo largo de un año. 
Tabla 1. Lista de las especies de orquídeas registradas a la venta en el mercado municipal Juan Sabines de Las Margaritas, Chiapas. Hábito de las especies: $\mathrm{E}=$ Epífita y T $=$ Terrestre. Categoría de riesgo de acuerdo a la NOM-059 (SEMARNAT-2010): P = En peligro de extinción, A $=$ Amenazada, $\mathrm{NE}=$ No Evaluada. Meses del año en los que cada especie fue registrada en el mercado: ENE $=$ Enero, FEB $=$ Febrero, $\mathrm{MAR}=$ Marzo, ABR $=$ Abril, MAY $=$ Mayo, JUN $=$ Junio, JUL $=$ Julio, AGO = Agosto, SEP $=$ Septiembre, OCT $=$ Octubre, NOV $=$ Noviembre, DIC $=$ Diciembre. Tamaño de flor y precio de cada especie. * Epidendrum sp. y Pleurothallis aff. correlli Luer, fueron especies acompañantes, se vendían junto a otras, por lo tanto, no se utilizaron en los análisis y no presentan datos sobre el tamaño de flores y el precio.

\begin{tabular}{|c|c|c|c|c|c|}
\hline Especie & Hábito & $\begin{array}{l}\text { Categoría } \\
\text { de riesgo }\end{array}$ & Mes & $\begin{array}{l}\text { Tamaño de flor } \\
\text { (diámetro en cm) }\end{array}$ & $\begin{array}{l}\text { Precio } \\
\text { (\$ MX.) }\end{array}$ \\
\hline Arpophyllum medium Rchb. f. & $\mathrm{E}$ & $\mathrm{NE}$ & NOV-DIC & 0.95 & 25 \\
\hline Aulosepalum hemichreum (Lindl.) Schltr. & $\mathrm{T}$ & $\mathrm{NE}$ & ABR-MAY & 1.1 & 10 \\
\hline Barkeria spectabilis Bateman ex Lindl. & $\mathrm{E}$ & NE & MAY & 6.7 & 15 \\
\hline Bletia purpurea (Lam.) DC. & $\mathrm{T}$ & $\mathrm{NE}$ & ENE, ABR-MAY & 1.2 & 10 \\
\hline Brassia maculata $\mathrm{R} . \mathrm{Br}$. & $\mathrm{E}$ & $\mathrm{NE}$ & MAY-JUN & 11.4 & 25 \\
\hline Brassia verrucosa Lindl. & E & NE & MAY-JUN & 10.2 & 20 \\
\hline Catasetum integerrimum Hook. & $\mathrm{E}$ & $\mathrm{NE}$ & MAY-JUN & 5.7 & 25 \\
\hline Chysis laevis Lindl. & $\mathrm{E}$ & $\mathrm{NE}$ & JUN & 9 & 30 \\
\hline Coelia macrostachya Lindl. & E & $\mathrm{NE}$ & $\mathrm{AGO}$ & 1.3 & 20 \\
\hline Coelia triptera (Sm.) G. Don ex Steud. & E & NE & DIC & 2.3 & 20 \\
\hline Cuitlauzina pulchella (Bateman ex Lindl.) Dressler \& N.H. Williams & $\mathrm{E}$ & $\mathrm{NE}$ & NOV-DIC & 5 & 15 \\
\hline $\begin{array}{l}\text { Cyrtopodium macrobulbon (La Llave \& Lex.) G.A. Romero \& } \\
\text { Carnevali }\end{array}$ & $\mathrm{T}$ & $\mathrm{NE}$ & MAR & 4.2 & 25 \\
\hline Dichaea glauca (Sw.) Lindl. & $\mathrm{E}$ & $\mathrm{NE}$ & JUL-AGO & 2.9 & 5 \\
\hline Dichaea muricatoides Hamer \& Garay & $\mathrm{E}$ & $\mathrm{NE}$ & JUN-AGO & 2.8 & 5 \\
\hline Domingoa purpurea (Lindl.) van den Berg \& Soto Arenas & E & $\mathrm{NE}$ & AGO-OCT & 0.7 & 5 \\
\hline Elleanthus cynarocephalus (Rchb. f.) Rchb. f. & $\mathrm{E}$ & $\mathrm{NE}$ & SEP & 2 & 10 \\
\hline Elleanthus graminifolius (Barb. Rodr.) Løjtnant & $\mathrm{E}$ & $\mathrm{NE}$ & DIC & 1.3 & 5 \\
\hline Encyclia ambigua (Lindl.) Schltr. & $\mathrm{E}$ & $\mathrm{NE}$ & ABR-MAY & 5.6 & 20 \\
\hline Encyclia cordigera (Kunth) Dressler & $\mathrm{E}$ & $\mathrm{NE}$ & FEB & 7.9 & 20 \\
\hline Encyclia diota (Lindl.) Schltr. & $\mathrm{E}$ & $\mathrm{NE}$ & MAR & 5.5 & 20 \\
\hline Encyclia hanburyi (Lindl.) Schltr. & $\mathrm{E}$ & $\mathrm{NE}$ & JUN & 5.4 & 15 \\
\hline Encyclia incumbens (Lindl.) Mabb. & $\mathrm{E}$ & $\mathrm{NE}$ & FEB-MAY & 5 & 20 \\
\hline Epidendrum alvarezdeltoroi Hágsater & $\mathrm{E}$ & $\mathrm{NE}$ & DIC & 4 & 5 \\
\hline Epidendrum chlorops Rchb. f. & $\mathrm{E}$ & $\mathrm{NE}$ & ENE & 3.9 & 10 \\
\hline Epidendrum cristatum Ruiz \& Pav. & $\mathrm{T}$ & $\mathrm{NE}$ & $\mathrm{ABR}$ & 4 & 15 \\
\hline Epidendrum radicans Pav. ex Lindl. & $\mathrm{T}$ & NE & DIC-ENE & 1.6 & 10 \\
\hline Epidendrum sp. & $\mathrm{T}$ & $\mathrm{NE}$ & ENE & - & - \\
\hline Gongora galeata (Lindl.) Rchb. f. & E & $\mathrm{NE}$ & NOV-DIC & 4.1 & 25 \\
\hline $\begin{array}{l}\text { Guarianthe aurantiaca (Bateman ex Lindl.) Dressler \& W.E. } \\
\text { Higgins }\end{array}$ & $\mathrm{E}$ & NE & DIC-ENE & 7.2 & 15 \\
\hline Isochilus carnosiflorus Lindl. & $\mathrm{E}$ & $\mathrm{NE}$ & JUN & 0.5 & 5 \\
\hline Jacquiniella cobanensis (Ames \& Schltr.) Dressler & $\mathrm{E}$ & $\mathrm{NE}$ & MAR-ABR & 0.6 & 5 \\
\hline Laelia anceps Lindl. & $\mathrm{E}$ & $\mathrm{NE}$ & AGO-OCT & 11.3 & 30 \\
\hline Laelia superbiens Lindl. & $\mathrm{E}$ & A & OCT-ENE & 15.6 & 45 \\
\hline Lockhartia oerstedii Rchb. f. & $\mathrm{E}$ & $\mathrm{NE}$ & MAY & 1.1 & 10 \\
\hline Lycaste aromatica (Graham) Lindl. & $\mathrm{E}$ & $\mathrm{NE}$ & FEB & 6 & 15 \\
\hline Lycaste cruenta (Lindl.) Lindl. & E & $\mathrm{NE}$ & NOV-DIC & 4.3 & 10 \\
\hline Lycaste skinneri (Bateman ex Lindl.) Lindl. & $\mathrm{E}$ & $\mathrm{P}$ & NOV-DIC & 14 & 100 \\
\hline Maxillaria elatior (Rchb.f.) Rchb. f. & $\mathrm{E}$ & $\mathrm{NE}$ & NOV & 5.6 & 20 \\
\hline
\end{tabular}




\begin{tabular}{|c|c|c|c|c|c|}
\hline Especie & Hábito & $\begin{array}{l}\text { Categoría } \\
\text { de riesgo }\end{array}$ & Mes & $\begin{array}{l}\text { Tamaño de flor } \\
\text { (diámetro en cm) }\end{array}$ & $\begin{array}{l}\text { Precio } \\
\text { (\$ MX.) }\end{array}$ \\
\hline Maxillaria pulchra (Schltr.) L.O. Williams ex Correl & $\mathrm{E}$ & $\mathrm{NE}$ & MAY-JUN & 4.9 & 10 \\
\hline Maxillaria variabilis Bateman ex Lindl. & $\mathrm{E}$ & $\mathrm{NE}$ & DIC & 3.3 & 10 \\
\hline Mormodes lineata Bateman ex Lindl. & E & NE & ENE-FEB & 5.6 & 45 \\
\hline Mormodes nagelii L.O. Williams & $\mathrm{E}$ & $\mathrm{NE}$ & MAY & 5.8 & 40 \\
\hline Oncidium endocharis Rchb. f. & $\mathrm{E}$ & A & DIC & 3.9 & 15 \\
\hline Oncidium leucochilum Bateman ex Lindl. & $\mathrm{E}$ & A & MAY-JUN y DIC & 3.4 & 10 \\
\hline Oncidium sotoanum R. Jiménez \& Hágsater & $\mathrm{E}$ & NE & DIC & 3.5 & 25 \\
\hline Oncidium sphacelatum Lindl. & $\mathrm{E}$ & $\mathrm{NE}$ & MAY-JUN & 3.9 & 15 \\
\hline Pleurothallis aff. correlli Luer & E & NE & DIC-ENE & - & - \\
\hline Pleurothallis antonensis L.O. Williams & $\mathrm{E}$ & $\mathrm{NE}$ & $\mathrm{AGO}$ & 1 & 10 \\
\hline Pleurothallis cardiothallis Rchb.f. & E & $\mathrm{NE}$ & DIC & 2.6 & 10 \\
\hline Prosthechea baculus (Rchb.f.) W.E. Higgins & $\mathrm{E}$ & $\mathrm{NE}$ & DIC-ENE & 5.1 & 10 \\
\hline Prosthechea cochleata (L.) W.E. Higgins & E & $\mathrm{NE}$ & DIC & 5.7 & 10 \\
\hline Rhynchostele bictoniensis (Bateman) Soto Arenas \& Salazar & $\mathrm{E}$ & $\mathrm{NE}$ & NOV-DIC & 4.8 & 20 \\
\hline Sobralia macrantha Lindl. & $\mathrm{T}$ & $\mathrm{NE}$ & JUN & 10.4 & 25 \\
\hline Sobralia xantholeuca B.S. Williams & $\mathrm{T}$ & $\mathrm{NE}$ & JUN & 10.2 & 30 \\
\hline Stanhopea aff. graveolens Lindl. & E & $\mathrm{NE}$ & JUL-GO & 14 & 20 \\
\hline Stanhopea aff. saccata Bateman & $\mathrm{E}$ & $\mathrm{NE}$ & AGO & 14.2 & 20 \\
\hline Stanhopea oculata (G.Lodd.) Lindl. & $\mathrm{E}$ & $\mathrm{NE}$ & JUL-AGO & 14.6 & 20 \\
\hline Stelis emarginata (Lindl.) Soto Arenas \& Solano & $\mathrm{E}$ & $\mathrm{NE}$ & ABR & 0.7 & 5 \\
\hline Stelis immersa (Linden \& Rchb.f.) Pridgeon \& M.W. Chase & $\mathrm{E}$ & $\mathrm{NE}$ & DIC & 1.1 & 10 \\
\hline Xylobium foveatum (Lindl.) G. Nicholson & $\mathrm{E}$ & $\mathrm{NE}$ & $\mathrm{AGO}$ & 2.2 & 15 \\
\hline
\end{tabular}

nicipal. Además, Guadalupe los Altos fue el ejido más lejano respecto al mercado (aprox. $60 \mathrm{~km}$; Figura 3).

La mayoría de los vendedores son agricultores-indígenas de la región, que colectan las plantas en los bosques alrededor de su ejido. Adicionalmente se encontró que la mayoría de los vendedores fueron mujeres $(86.6 \%) ; 26.6 \%$ se dedicaban a otras actividades en el mercado y $73.4 \%$ se dedicaban exclusivamente a la venta de orquídeas. Los análisis de correlación indicaron alta colinealidad entre el precio y el tamaño de la flor $(r=0.67, p<0.01$; Tabla 1$)$.

\section{Discusión}

El número de especies registradas a la venta en el mercado visitado representa $18 \%$ de la riqueza orquideológica reportada para la RTP El Momón-Montebello (Soto-Arenas 2001) y $8.3 \%$ del total estatal (Beutelspacher \& Moreno 2018). La riqueza de orquídeas registrada en venta en el mercado de Las Margaritas, resulta ser de las más altas, hasta el momento, documentadas en un mercado tradicional, solo superada por la reportada para los mercados de la ciudad de Xalapa, Veracruz: 167 especies de orquídeas (Flores-Palacios \& Valencia-Díaz 2007), pero es mayor para especies comercializadas en mercados de la Ciudad de México: 22 especies (Munguía-Lino et al. 2010), del sur del Estado de México: 6 especies (Emeterio-Lara et al. 2016), de Tlaxiaco, Oaxaca:
36 especies (Cruz-García et al. 2015) y de Berriozábal, Chiapas 50 especies (Martínez-Meléndez et al. 2016).

Los géneros más comercializados en este mercado, concuerda con las observaciones previas, que indican que son los más comercializados en mercados de México (Cruz-García et al. 2015, Emeterio-Lara et al. 2016), y es debido al precio accesible en la venta ilegal y la vistosidad de las flores (Emeterio-Lara et al. 2016, Jiménez-López et al. 2019). Los precios de venta en este mercado van desde \$5 MXN hasta $\$ 100 \mathrm{MXN}$. Algunas especies como L. anceps se venden a un precio similar $( \pm \$ 30 \mathrm{MXN})$ en Oaxaca, en tanto que el precio de Cyrtopodium macrobulbon La Llave \& Lex. (\$25 MXN) es mucho mayor en los mercados de Oaxaca, donde alcanza los \$138 MXN (Cruz-García et al. 2015). En general, el precio de las especies de Stanhopea J. Frost ex Hook. es similar a los precios en mercados del sur del estado de México, entre \$20 y \$30 MXN (Emeterio-Lara et al. 2016). Por el contrario, los precio en la venta legal de orquídeas por empresas mexicanas, se elevan en la mayoría de los casos en más de $100 \%$ con respecto al precio con especies de venta ilegal en mercados tradicionales, por ejemplo: L. anceps $\$ 320$ vs. $\$ 30 \mathrm{MXN}$, Brassia verrucosa Lindl. \$350 vs. \$20 MXN y Encyclia cordigera (Kunth) Dressler \$250 vs. \$20 MXN (venta legal vs. venta ilegal, respectivamente, Orquídeas río verde 2019). Estas diferencias pondrían en serios problemas la comercialización de orquídeas desde el punto de vista del 


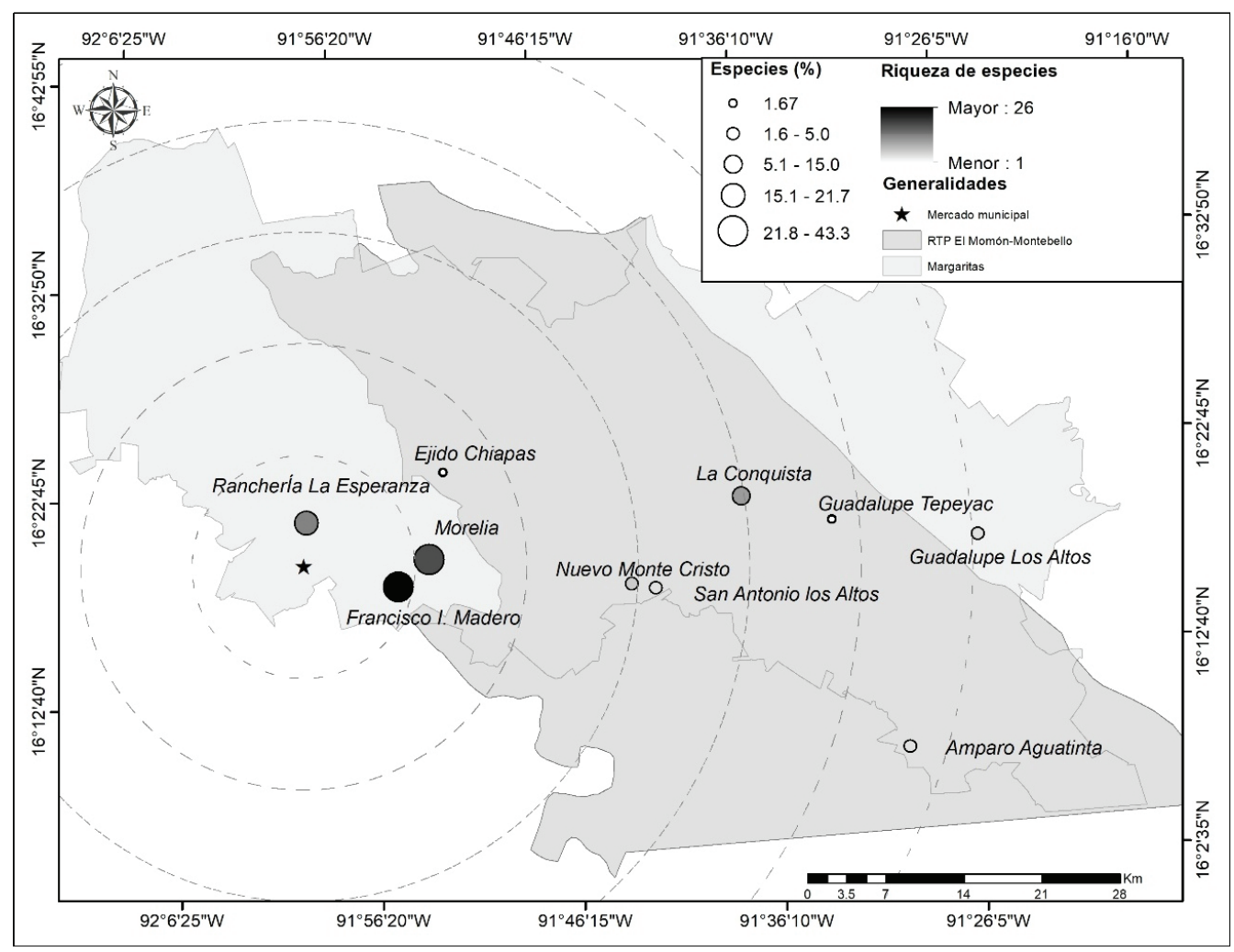

Figura 3. Número de especies, porcentaje y lugares de extracción (ejidos) para la venta de orquídeas en el mercado. Los círculos con líneas discontinuas (radios de $10 \mathrm{~km}$ ) corresponden a la distancia entre el mercado y los ejidos donde se extraen las plantas.

marco legal, y su implementación a nivel local y regional en esta zona estaría destinado al fracaso.

En el mercado de Las Margaritas se sabe que, del total de especies, 54 se compran con fines de colección o como plantas de ornato, y las inflorescencias para adornos temporales. Cuatro de esas especies están protegidas por las leyes mexicanas (Lycaste skinneri, Laelia superbiens, Oncidium endocharis y O. leucochilum). En tanto, L. anceps y Cuitlauzina pulchella, las dos especies registradas con mayor frecuencia de venta durante el año de estudio, no están consideradas en riesgo. El precio y la vistosidad de las flores de una orquídea terrestre fue menor al de una epífita. Por lo tanto, el comercio para estas especies (en peligro y las de mayor frecuencia) es motivado por la demanda de las plantas para uso religioso como adornos temporales, y debido también a que su floración coincide con las festividades religiosas, además del atractivo visual de sus flores (Solano-Gómez et al. 2010, Jiménez-López et al. 2019). En México, las poblaciones de Lycaste skinneri, Laelia superbiens y Oncidium endocharis sólo se conocen del estado de Chiapas (Halbinger \& Soto 1997, Jiménez-Machorro 2002, Cabrera 2006; Soto-Arenas \& Solano 2007) y son constantemente comercializadas, al menos a nivel regional (Martínez-Meléndez et al. 2016, Jiménez-López et al. 2019), y para el caso de L. skinneri y L. superbiens fueron las especies con mayor abundancia y los precios más altos en venta, en épocas decembrinas, en este mismo mercado (Jiménez-López et al. 2019). Por lo tanto, debido a que las poblaciones de estas especies están sujetas a la extracción año con año, y sumado a que su hábitat está siendo afectado por la práctica de actividades agropecuarias y el desarrollo urbano (Soto-Arenas et al. 2007), la conservación de estas poblaciones está en grave riesgo. Se sabe que el comercio local ilegal pone en riesgo la diversidad genética y reduce el tamaño de las poblaciones, situación que ya se ha observado en Laelia speciosa (Kunth) Schltr., en Michoacán (Rojas-Méndez et al. 2017).

Los periodos de mayor venta de orquídeas coinciden con los periodos en que la mayoría de las especies comercializadas se encuentran en floración (diciembre-enero y mayojunio) un patrón ya reportado por Cruz-García et al. (2015), y que corresponden con las temporadas de mayor floración para la orquideoflora que ha sido registrada en la región de Montebello (Soto-Arenas 2001). Los periodos de menor venta (septiembre-octubre), corresponden con una menor disponibilidad de ejemplares en floración en su hábitat para aquellas especies de flores vistosas, las cuales tienen mayor demanda en el mercado y, además, es también el tiempo en el cual las lluvias en la región son intensas y dificultan el trabajo de recolecta de orquídeas.

Los ejidos Francisco I. Madero y Morelia albergan bosques de encino, bosques de pino-encino y relictos de bosque mesófilo de montaña, de donde se extrae la mayoría de las 
especies registradas en el mercado visitado. Estas comunidades se encuentran muy próximas a la cabecera de Las Margaritas y cuentan con servicios de transporte interurbano, lo cual facilita el transporte de los vendedores al mercado. Una situación similar se observó en los vendedores de orquídeas en el mercado de Tlaxiaco, Oaxaca (Cruz-García et al. 2015) y de la misma región (Jiménez-López et al. 2019), lo que podría indicar que la cercanía al mercado y el acceso con transporte urbano está relacionada con llevar una mayor riqueza de especies para la venta. La mayoría de los bosques de donde se extraen las orquídeas comercializadas en Las Margaritas se encuentran en la RTP El Momón-Montebello, la cual alberga la mayor diversidad alfa de orquídeas en México (Soto-Arenas 2001). Los bosques predominantes en esta región son los de coníferas, encinos y mesófilo de montaña (Hágsater et al. 2005), los cuales son hábitats favorables para las orquídeas, incluidas aquellas que fueron más las frecuentemente comercializadas. La extracción de plantas para el comercio local, la pérdida y transformación del hábitat y la presencia de incendios ha ocasionado que muchas especies de orquídeas presenten una reducción en sus tamaños poblacionales en los bosques de la RTP El Momón-Montebello y que incluso, algunas de ellas hayan llegado a la extinción a nivel nacional, pues sus poblaciones en el país sólo se conocían en esta región (Soto-Arenas et al. 2007, Solano-Gómez et al. 2012), como Dichaea tuerckheimii Schltr., Epidendrum pansamalae Schltr., Jacquiniella gigantea Dressler, Salazar \& García Cruz, Lepanthes minima Salazar \& Soto Arenas, L. stenophylla Schltr., L. yunckeri Ames, Lycaste dowiana Endress \& Rchb. f., L. lasioglossa Rchb. y Specklinia samacensis (Ames) Pridgeon \& M.W. Chase. En la medida de lo posible, realizar un censo de las poblaciones de las especies con estatus de riesgo en la legislación mexicana y que tienen una mayor demanda en el mercado (Lycaste skinneri, Laelia anceps, L. superbiens y Oncidium leucochilum), lo cual ayudaría para tomar acciones específicas para establecer políticas para su conservación y manejo.

Se observó que las mujeres adultas (33.2 años en promedio) eran las que vendían las orquídeas más vistosas a mayores precios, fenómeno que se ha observado para otros mercados de México (Emeterio-Lara et al. 2016, JiménezLópez et al. 2019). La mayoría de los vendedores en el mercado de Las Margaritas solo comercializan orquídeas, aunque se observó lo contrario en un estudio realizado en 2016 en épocas decembrinas (Jiménez-López et al. 2019). Este tipo de comercio parece ser una singularidad de este mercado, pues otros estudios mencionan que los vendedores de orquídeas complementan esta actividad vendiendo productos de la milpa (frutas, verduras), plantas medicinales, animales de granja, etc. (Flores-Palacios \& Valencia-Díaz 2007, Cruz-García et al. 2015, Emeterio-Lara et al. 2016, Jiménez-López et al. 2019). Una explicación para lo que sucede en el mercado de Las Margaritas se debe a que aquí, la principal actividad de los vendedores de orquídeas no está relacionada con el trabajo en campo, sino aquellas relacionadas con el sector terciario o de servicios (e.g., cobro del envío de remesas de sus familiares, trámites de INE, administrativos en hacienda y presidencia municipal, etc.). Así, para los vendedores de Las Margaritas el comercio de orquídeas puede ser una actividad complementaria a otra que nada tiene que ver con el sector primario (agrícola).

Nuestros resultados corroboraron la hipótesis planteada, el precio de las orquídeas está determinado por ciertos atributos de la planta, en este caso, la vistosidad de la flor de la especie, sugiriendo una posible relación entre el tamaño de la flor y el volumen de ventas para las orquídeas silvestres comercializadas en mercados tradicionales mexicanos, aunque solo de manera descriptiva (Emeterio-Lara et al. 2016), este trabajo y otro en la misma región están determinando la misma tendencia (Jiménez-López et al. 2019). Aunque se sabe que existe una relación entre la oferta y la demanda (Flores-Palacios \& Valencia-Díaz 2007, Cruz-García et al. 2015, Jiménez-López et al. 2019), este tipo de relación (precio vs. vistosidad) estaría siendo una variable que podría afectar el patrón de oferta-demanda, por lo que se exhorta a seguir trabajando con este tipo de variables en estudios de comercio de plantas en mercados tradicionales, y tomar la vistosidad de la flor para diseñar un posible programa de gestión para las orquídeas comercializadas en mercados tradicionales de México (Jiménez-López et al. 2019).

En el mercado estudiado, el comercio de orquídeas se realiza prácticamente todo el año, está basado en la extracción de ejemplares silvestres que crecen en bosques bajo protección federal y se realiza sin ningún tipo de regulación. Tanto los sitios de extracción como los de venta están en la misma región, por lo que es un comercio local, donde, además, el vendedor es también el colector de las orquídeas. En virtud de lo anterior, es necesario organizar talleres con los vendedores para informar sobre el problema que representa esta actividad y de su posible realización desde el marco legal y al mismo tiempo conservar el recurso. La conservación del recurso también involucra implementar prácticas de manejo para las orquídeas, por ejemplo, Unidades de Manejo Ambiental (UMA) o viveros rústicos, con la inclusión de las personas residentes de aquellas comunidades donde se ha observado extracción de orquídeas silvestres.

\section{Agradecimientos}

A Rosalinda López López por la ayuda en las entrevistas y la mayoría de los recorridos en el mercado. A Jorge A. Meave, Martha Martínez Gordillo y dos revisores anónimos por las sugerencias para enriquecer este manuscrito. A Carlos Rommel Beutelspacher, Eric Hágsater y Gerardo Salazar, por la ayuda en la identificación de las especies. A Candelario Peralta por la elaboración de los mapas y Jonathan V. Solórzano por la traducción del resumen.

\section{Literatura citada}

Arellanes Y, Casas A, Arellanes A, Vega E, Blancas J, Vallejo M, Torres I, Rangel-Landa S, Moreno AI, Solís L, PérezNegrón E. 2013. Influence of traditional markets on plant management in the Tehuacán Valley. Journal of Ethnobiology and Ethnomedicine 9: 38.

DOI: https://doi.org/10.1186/1746-4269-9-38 
Arriaga-Cabrera L, Espinosa-Rodríguez J, Aguilar-Zúñiga C, Martínez-Romero E, Gómez-Mendoza L, Loa-Loza E. 2000. Regiones Terrestres Prioritarias de México. México, D.F.: Comisión Nacional para el Conocimiento y Uso de la Biodiversidad. ISBN 970-9000-16-0

Beutelspacher BCR, Moreno MI. 2018. Las Orquídeas de Chiapas. Ciudad de México: Instituto Chinoín AC. ISBN: 978607-7597-06-3

Bye RA, Linares E. 1983. The role of plants found in the Mexican markets and their importance in ethnobotanical studies. Journal of Ethnobiology 3: 1-13.

Cabrera T. 2006. Ficha técnica de Lycaste skinneri. Cuarenta y ocho especies de la flora de Chiapas incluidas en el PROYNOM-059-ECOL-2000. <http://www.conabio.gob.mx/conocimiento/ise/fichasnom/Lycasteskinneri00.pdf> (acceso junio 9, 2019).

Cruz-García G, Lagunes-Rivera L, Chávez-Ángeles MG, Solano-Gómez R. 2015. The wild orchid trade in a Mexican local market: diversity and economics. Economic Botany 20: 1-15. DOI: https://doi.org/10.1007/s12231-015-9321-z

Emeterio-Lara A, Palma-Linares V, Vázquez-García LMJ, Mejía-Carranza J. 2016. Usos y comercialización de orquídeas silvestres en la región sur del Estado de México. Polibotánica 42: $197-214$.

DOI: http://dx.doi.org/10.18387/polibotanica.42.10

Farfán-Heredia B, Casas A, Moreno-Calles AI, García-Frapolli E, Castilleja A. 2018. Ethnoecology of the interchange of wild and weedy plants and mushrooms in Phurépecha markets of Mexico: economic motives of biotic resources management. Journal of Ethnobiology and Ethnomedicine 14: 5. DOI: https://doi.org/10.1186/s13002-018-0205-z

Flores-Palacios A, Valencia-Díaz S. 2007. Local illegal trade reveals unknown diversity and involves a high species richness of wild vascular epiphytes. Biological Conservation 136: 372-387. DOI: https://doi.org/10.1016/j.biocon.2006.12.017

Hágsater E, Soto-Arenas MA, Salazar-Chávez GA, JiménezMachorro R, López-Rosas MA, Dressler RL. 2005. Las Orquídeas de México. Ciudad de México: Instituto Chinoín. ISBN-13: 9789687889078

Halbinger F, Soto M. 1997. Laelias of Mexico. Orquidea (Mex. City), n.s. 15; 1-60.

Jiménez-López DA, Martínez-Meléndez N, Roblero-Velasco RJ. 2015. Comercio de orquídeas silvestres en Las Margaritas, Chiapas, México: Boletín de la AMO. Septiembre-Octubre.

Jiménez-López DA, Solano R, Peralta-Carreta C, Solórzano JV, Chávez-Ángeles MG. 2019. Species richness may determine the income from illicit wild orchid trading in traditional markets in Mexico. Economic Botany 73: 171-186. DOI: https://doi.org/10.1007/s12231-019-09460-5

Jiménez-Machorro R. 2002. Oncidium endocharis Rchb.f., Gard. Chron. n. s. 21: 2006. 1884. In: Hágsater E, Soto MM. (eds.). Icones Orchidaceae (Mexico). Fasc. 5-6. Orchids of Mexico. Partes 2-3. Herbario AMO. Pl. 630.

Linares E, Bye R. 2016. Traditional markets in Mesoamerica. A mosaic of history and traditions. In: Lira R, Casas A, Blancas J, eds. Ethnobotany of Mexico: Interactions of People and Plants in Mesoamerica, pp. 151-177, New York: Springer. eBook ISBN 978-1-4614-6669-7;

DOI: https://doi.org/10.1007/978-1-4614-6669-7
Martínez-Meléndez N, López-Pereyra RM, Escobar-Castellanos MA. 2016. Plantas de terraza: las epífitas de Berriozábal, Chiapas, México. Desde el Herbario CICY 8: 69-72.

Mondragón D. 2009. Population viability analysis for Guarianthe aurantiaca, an ornamental epiphytic orchid harvested in southeast México. Plant Species Biology 24: 35-41. DOI: https://doi.org/10.1111/j.1442-1984.2009.00230.x

Mondragón D. 2015. La comercialización navideña de bromelias epífitas en la ciudad de Oaxaca, México. Etnobiología 6: $24-28$.

Müllerried FKG. 1957. La Geología de Chiapas. México: Gobierno del Estado de Chiapas.

Munguía-Lino G, Vázquez-García LM, López-Sandoval JA. 2010. Plantas silvestres ornamentales comercializadas en los mercados de la flor de Tenancingo y Jamaica, México. Polibotánica 29: 281-308.

Orquídeas río verde. 2019. Catalogo orquídeas primavera 2019. http://wp.orquideas.com.mx/wp-content/uploads/2011/10/ Micelanea-especies-enero-2019.pdf (acceso junio 4, 2019).

R Core Team. 2016. R: A language and environment for statistical computing. R Foundation $<$ https://www.r-project.org/> (Acceso agosto 20, 2018).

Reuter A. 2009. Reflexiones sobre el comercio de vida silvestre en México. Biodiversitas 84: 11-15.

Rojas-Méndez KJ, Peñaloza-Ramírez JM, Rocha-Ramírez V, Cortés-Palomec A, McCauley RA, Oyama K. 2017. Massive extraction of the orchid Laelia speciosa (HBK) Schltr. for trading in local markets affect its population genetic structure in a fragmented landscape in central Mexico. Tropical Conservation Science 10:

DOI: https://doi.org/10.1177/1940082917693235

Rzedowski J. 1978. Vegetación de México. Limusa, México, D.F. ISBN: 978-968-1800-0-24.

SEGOB [secretaría de Gobernación]. 2010. Enciclopedia de los municipios y delegaciones de México, Estado de Chiapas. Instituto para el Federalismo y el Desarrollo Municipal (INAFED). Chiapas, México. http://www.inafed.gob. mx/work/enciclopedia/EMM07chiapas/ (Acceso diciembre 20, 2014).

SEMARNAT [Secretaría del Medio Ambiente y Recursos Naturales]. 2003. Norma Oficial Mexicana-005-SEMARNAT1997 que establece los procedimientos, criterios y especificaciones para realizar el aprovechamiento, transporte y almacenamiento de corteza, tallos y plantas completas de vegetación forestal. Diario Oficial de la Federación, 23 de abril de 2003.

SEMARNAT. 2010. Norma Oficial Mexicana NOM-059-SEMARNAT-2010, Protección ambiental - Especies nativas de México de flora y fauna silvestres - Categorías de riesgo y especificaciones para su inclusión, exclusión o cambio - Lista de especies en riesgo. Diario Oficial de la Federación. 2da Sección, 30 de diciembre de 2010.

SEMARNAT. 2014. Ley General del Equilibrio Ecológico y la Protección al Ambiente. Diario Oficial de la Federación. 28 de enero de 1988.

SEMARNAT. 2018a. Ley General de Vida Silvestre. Diario Oficial de la Federación.19 de enero de 2018. 
SEMARNAT. 2018b. Ley General de Desarrollo Forestal Sustentable. Diario Oficial de la Federación. 5 de junio de 2018.

Shackleton CM. 2001. Re-examining local and market-orientated use of wild species for the conservation of biodiversity. Environmental Conservation 28: 270-278. DOI: https://doi.org/10.1017/S0376892901000285

Solano-Gómez R, Cruz-Lustre G, Martínez-Feria A, LagunezRivera L. 2010. Plantas utilizadas en la celebración de la semana santa en Zaachila, Oaxaca, México. Polibotánica 29: $263-279$.

Solano-Gómez R, Soto-Arenas MA, Hágsater E. 2012. Riesgo de extinción y patrones de pérdida de diversidad en orquídeas mexicanas. In: Téllez-Velasco MA. ed. Conservación de Orquídeas en México, pp. 221-229, México, D.F.:Instituto de Biología, Universidad Nacional Autónoma de México.

Soto-Arenas MA. 2001. Diversidad de orquideas en la región El Momón-Margaritas-Montebello, Chiapas, México. México D.F.: Instituto Chinoin AC. Herbario de la Asociación Mexicana de Orquideología AC. Informe final SNIB-CONABIO proyecto No. R225. http://www.conabio.gob.mx/insti-
tucion/proyectos/resultados/InfR225.pdf (Acceso enero 4, 2015).

Soto-Arenas MA, Solano R. 2007. Ficha técnica de Oncidium endocharis. In: Soto-Arenas MA. Información Actualizada sobre las Especies de Orquideas del PROY-NOM-ECOL2000, pp 1-6, México D.F.: Instituto Chinoín A.C. y Herbario de la Asociación Mexicana de Orquideología, A.C. http:// www.conabio.gob.mx/conocimiento/ise/fichasnom/Oncidiumendocharis00.pdf (Acceso febrero 8, 2015)

Soto-Arenas MA, Solano R, Hágsater E. 2007. Risk of extinction and patterns of diversity loss in Mexican orchids. Lankesteriana 7:114-121.

Villamar AA. 2016. El estudio etnobioecológico de los tianguis y mercados en México. Etnobiología 14: 38-46.

Villaseñor JL. 2016. Checklist of the native vascular plants of Mexico. Revista Mexicana de Biodiversidad 87: 559-902. DOI: https://doi.org/10.1016/j.rmb.2016.06.017

Vodouhê FG, Coulibaly O, Greene C, Sinsin B. 2009. Estimating the local value of non-timber forest products to Pendjari Biosphere Reserve Dwellers in Benin. Economic Botany 63: 397-412. DOI: https://doi.org/10.1007/s12231-009-9102-7

Editor de sección: Martha Martínez-Gordillo

Contribución de los autores: DAJL (https://orcid.org/0000-00015270-9191) concibió la idea, realizó los análisis y escribió el artículo, EAPG y NMM analizaron la información y contribuyeron a escribir el artículo, RS analizó los resultados y sintetizó la información, todos los autores contribuyeron a la versión final del escrito. 